\title{
Erratum to Protective intraoperative ventilation with higher versus lower levels of positive end-expiratory pressure in obese patients (PROBESE): study protocol for a randomized controlled trial
}

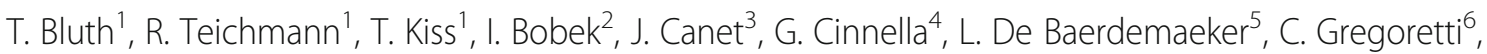 \\ G. Hedenstierna', S. N. Hemmes ${ }^{8}$, M. Hiesmayr ${ }^{9}$, M. W. Hollmann ${ }^{8}$, S. Jaber ${ }^{10}$, J. G. Laffey ${ }^{11}$, M. J. Licker ${ }^{12}$, \\ K. Markstaller ${ }^{13}$, I. Matot ${ }^{14}$, G. Müller ${ }^{15}$, G. H. Mills ${ }^{16}$, J. P. Mulier ${ }^{17}$, C. Putensen ${ }^{18}$, R. Rossaint ${ }^{19}$, J. Schmitt ${ }^{15}$, \\ M. Senturk ${ }^{20}$, A. Serpa Neto ${ }^{21}$, P. Severgnini ${ }^{22}$, J. Sprung ${ }^{23}$, M. F. Vidal Melo ${ }^{24}$, H. Wrigge ${ }^{25}$, M. J. Schultz ${ }^{26}$, \\ P. Pelosi ${ }^{27}$, Marcelo Gama de Abreu ${ }^{1 *}$ and for the PROBESE investigators, and the PROtective VEntilation Network \\ (PROVEnet) and on behalf of the Clinical Trial Network of the European Society of Anaesthesiology (ESA)
}

\section{Erratum}

In the original publication [1] one author name was not correct.

Wrong: Miro, Zupcic

Correct: Zupcic, Miro

\begin{abstract}
Author details
'Department of Anesthesiology and Intensive Care Medicine, Pulmonary Engineering Group, University Hospital Carl Gustav Carus, Dresden, Germany. ${ }^{2}$ Aneszteziológiai és Intenzív Terápiás Klinika, Semmelweis Egyetem, Budapest, Hungary. ${ }^{3}$ Department of Anesthesiology, Hospital Universitari Germans Trias I Pujol, Badalona, Spain. ${ }^{4}$ Department of Anesthesiology and Intensive Care Medicine, University of Foggia, Foggia, Italy. ${ }^{5}$ Department of Anesthesiology, Ghent University Hospital, Ghent, Belgium. ${ }^{6}$ Department of DIBIMED, Policlinico P. Giaccone, Palermo, Italy. ${ }^{7}$ Department of Medical Sciences, Section of Clinical Physiology, University Hospital, Uppsala, Sweden. ${ }^{8}$ Department of Anesthesiology \& Laboratory of Experimental Intensive Care and Anesthesiology (L.E.I.C.A), Academic Medical Center, University of Amsterdam, Amsterdam, The Netherlands. 'Division Cardiac-, Thoracic-, Vascular Anesthesia and Intensive Care, Medical University of Vienna, Vienna, Austria. ${ }^{10}$ Department of Critical Care Medicine and Anesthesiology (SAR B), Saint Eloi University Hospital, Montpellier, France. ${ }^{11}$ Department of Anesthesia and Critical Care Medicine, Saint Michael's Hospital, and Departments of Anesthesia, Physiology and Interdepartmental division of Critical Care Medicine, University of Toronto, Toronto, Canada. ${ }^{12}$ Department of Anesthesiology, Pharmacology \& Intensive Care, University Hospital Geneva, Geneva, Switzerland. ${ }^{13}$ Department of Anesthesiology and Intensive Care Medicine, Medical University, Vienna, Austria. ${ }^{14}$ Department of Anesthesiology and Critical Care, Tel Aviv Medical Center, Sackler School of Medicine, Tel Aviv University, Tel Aviv, Israel. ${ }^{15}$ Center for Evidence-based Healthcare, University Hospital and Medical Faculty Carl Gustav Carus, Dresden, TU, Germany. ${ }^{16}$ Operating Services, Critical Care and Anaesthesia
\end{abstract}

(OSCCA), Sheffield Teaching Hospitals and University of Sheffield, Sheffield, UK. ${ }^{17}$ Department of Anesthesiology, AZ Sint Jan Brugge-Oostende AV, Brugge, Belgium. ${ }^{18}$ Department of Anesthesiology and Intensive Care Medicine, University of Bonn, Bonn, Germany. ${ }^{19}$ Department of Anesthesiology, University of Aachen, Aachen, Germany. ${ }^{20}$ Department of Anesthesiology and Intensive Care Medicine, Istanbul Medical Faculty, University of Istanbul, Istanbul, Turkey. ${ }^{21}$ Department of Critical Care Medicine, Hospital Israelita Albert Einstein, and Program of Post-Graduation, Research and Innovation, Faculdade de Medicina do ABC, São Paulo, Brazil. ${ }^{22}$ Department of Biotechnology and Sciences of Life, University of Insubria, ASST-settelaghi Ospedale di Cricolo e Fondazione Macchi, Varese, Italy.

${ }^{23}$ Department of Anesthesiology, Mayo Clinic, Rochester, MN, USA.

${ }^{24}$ Department of Anesthesia, Critical Care and Pain Medicine, Massachusetts General Hospital -Harvard Medical School, Boston, MA, USA. ${ }^{25}$ Department of Anesthesiology and Intensive Care Medicine, University of Leipzig, Leipzig, Germany. ${ }^{26}$ Department of Intensive Care \& Laboratory of Experimental Intensive Care and Anesthesiology (L.E.I.C.A), Academic Medical Center, University of Amsterdam, Amsterdam, The Netherlands. ${ }^{27}$ Department of Surgical Sciences and Integrated Diagnostics, IRCCS AOU San Martino, IST, University of Genoa, Genoa, Italy.

Received: 17 May 2017 Accepted: 17 May 2017

Published online: 01 June 2017

Reference

1. Protective intraoperative ventilation with higher versus lower levels of positive end-expiratory pressure in obese patients (PROBESE): study protocol for a randomized controlled trial. Trials 2017;18:202. doi: 10.1186/s13063017-1929-0

* Correspondence: mgabreu@uniklinikum-dresden.de

'Department of Anesthesiology and Intensive Care Medicine, Pulmonary

Engineering Group, University Hospital Carl Gustav Carus, Dresden, Germany 